\title{
Revisión de los programas para la inclusión educativa con niños con necesidades educativas especiales
}

\section{Review of programs for educational inclusion with children with special educational needs}

\author{
Zila Esteves Fajardo. \\ Universidad de Guayaquil, Ecuador \\ Norma Gárces Gárces. \\ Universidad de Guayaquil, Ecuador
}

Andrés Chávez

Universidad Internacional del Ecuador, Ecuador

Autor para correspondencia: jbalas@ups.edu.ec; anchavezer@uide.edu.ec; acedeno@ups.edu.ec Fecha de recepción: 10 de Septiembre de 2017 - Fecha de aceptación: 15 de Noviembre de 2017

Resumen: La innovación estética dentro de las unidades educativas de tercer nivel, demanda de una organización de recursos físicos, materiales y financieros, que solamente se los puede hacer desde la administración. La inclusión social de los niños merece un análisis de los diferentes contextos que llevará a tener un criterio muy claro del origen ambiental y genético de muchas de las realidades que se está viviendo y cómo el docente en los ámbitos de la educación ecuatoriana, incluirá en esos planes, las diferentes estrategias que habrá que tener en el aula para que se produzca esa rehabilitación básica. En el cerebro de las personas con Asperger, el 70\% de la comunicación entre las personas es el lenguaje no verbal, el 30\% se lo hace en forma literal, los de Asperger mantienen el 30\% de la forma literal. El Asperger es no visible, pero poseen un sistema repetitivo, ellos no miran a los ojos. Por ello se ha elaborado una encuesta con un trabajo de campo para con estudiantes de la Escuela de Párvulos de tercer y cuarto curso de la Universidad de Guayaquil.

Palabras claves: asperger; estética; autismo; genética

\begin{abstract}
The aesthetic innovation within the educational units of the third level, demands an organization of physical, material and financial resources, which can only be done by the administration. The social inclusion of children deserves an analysis of the different contexts that will lead to a very clear criterion of the environmental and genetic origin of many of the realities that are being lived and how the teacher in the fields of Ecuadorian education, will include those plans, the different strategies that must be taken in the classroom for this basic rehabilitation to take place. In the brain of people with Asperger, 70\% of communication between people is non-verbal language, 30\% is done literally, Asperger's maintain 30\% of the literal form. The Asperger is not visible, but they have a repetitive system, they do not look in the eyes. For this reason, a survey has been prepared with fieldwork for students of the third and fourth grade Párvulos School of the University of Guayaquil. Key words: asperger; aesthetics; autism; genetics
\end{abstract}




\section{Introducción}

La innovación artística dentro de las unidades educativas de tercer nivel, demanda de una organización de recursos físicos, materiales y financieros, que solamente se los puede hacer desde la administración educativa. La inclusión social en todos los ámbitos de la educación ecuatoriana, ha creado un sistema de insertar al niño con problemas específicos y muchas veces somáticos, pero no incluye en esos planes, las diferentes estrategias que habrá que tener en el aula para que se produzca esa rehabilitación básica.

En los niños con autismo, la principal teoría es que es causado por el tema de la vacuna y se dice que el mercurio está en estas vacunas y como es tóxico provoca autismo; pero lo que sucede es que va en aumento y no se suprime el proceso. La dieta libre de gluten y de caseína es la dieta que debe tener un niño con autismo, evitar el pan, la pizza, la galleta. Cuando el gluten entra al cuerpo se pega al cuerpo se pega y ataca al cuerpo. La caseína que tiene la leche, es lo que hace daño al niño autista al tener residuos de mercurio en su cuerpo. En la flora intestinal puede estar la calidad de la salud de una persona, es como su segundo cerebro. (Williams, 1995; Thomas, 2003; Szatmari, 2006).

Hace 72 años, el pediatra Hans Asperger, estudio en niños que presentaban conducta repetitiva y dificultades para socializar con los demás, y determinó que era una buena condición neurobiológica. En el cerebro de las personas con Asperger, el 70\% de la comunicación entre las personas es el lenguaje no verbal, el 30\% se lo hace en forma literal, los de Asperger mantienen el $30 \%$ de la forma literal. El Asperger es no visible, pero poseen un sistema repetitivo, ellos no miran a los ojos (Attwood, 2007; Wing, 1981).

\section{Objetivo General}

Determinar las características del autismo y el Asperger mediante un análisis bibliográfico para llegar a establecer estrategias de conciencia psicológica.

\section{Objetivos específicos}

$\checkmark$ Establecer las pautas de comportamiento que debe manejar el docente.

$\checkmark$ Desarrollar estrategias de inteligencia emocional para niños con NEE

\section{Justificación}

La zona cerebral de los con Asperger tienen muy desarrollada su zona cerebral, ellos pueden recordar y dibujar objetos con una buena memoria fotográfica, por lo que la maestra debe trabajar con ellos: darle énfasis en lo literal, en evaluar de forma que sea más visible para ellos rasgos de las cosas por su gran capacidad desarrollada en la memoria visual. La gestión de los recursos por lo tanto se debería hacerlo hacia cartulinas, elementos de diferentes colores, a detallar aspectos o categorías de los objetos adaptados a la edad de los niños. 


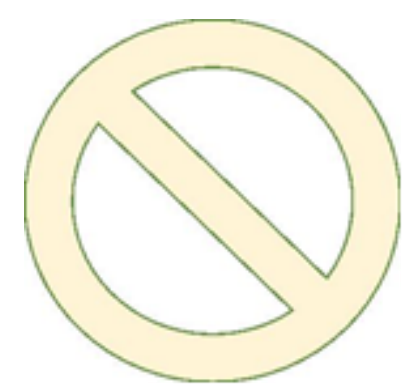

Un Asperger un niño puede seguir la ruta graficada varias veces, lo mismo un autista, tanto lo que está de amarilla como si los medio círculos también se puede desarrollar actos repetitivos. No es culpa de ellos. Ellos a veces ni se dan cuenta de que los miran.

Un niño Asperger posee también categorías comparativas como el hecho de no gustarle perder un autobús o un tren. Muchos de ellos no soportan los fracasos. La capacidad de disfrutar de la elegancia de la música de estos niños, es increíble.

Entre los signos están la mirada hacia abajo, son personas rutinarias, se molestan cuando algo sale de la rutina. Al no tener amigos, ellos se crean un mundo imaginario. Al no poder relacionarse con la gente, se crean un mundo imaginario y que los obliga a hablar solos y lo exteriorizan pero solos. Hablar solos, sus diálogos de otros personajes o personas, se ve por tanto interrumpida la socialización recíproca, en ese cruzar del mundo interior hacia el otro, la alteridad, característica de una inteligencia social normaloide, con el Asperger se ve interrumpida, y ellos no lo notan así. A partir de esa realidad, existen niños que han desarrollado sin embargo grandes habilidades a pesar de que lo poseen. Como que el poco desarrollo de otras habilidades, les ha permitido alcanzar niveles especiales en aspectos (Hall, 2003; Romanowski \& Kirby, 2005).

\section{Metodología}

En base al análisis de niños con NEE se elaboran seis escenarios para ver el desarrollo y comportamiento, junto con la evolución de los niños en los siguientes escenarios:

1. Colllage

2. Teatro

3. Músicoterapia

4. Danza

5. Programas informáticos de cuentos para niños.

6. Rutas en las cuales el niño de A, debe ir a B, luego a C, y D. En esas rutas al niño se le medirá la coordinación y la ubicación de materiales que les permita apreciar la belleza y mejorar su coordinación y lógica. 


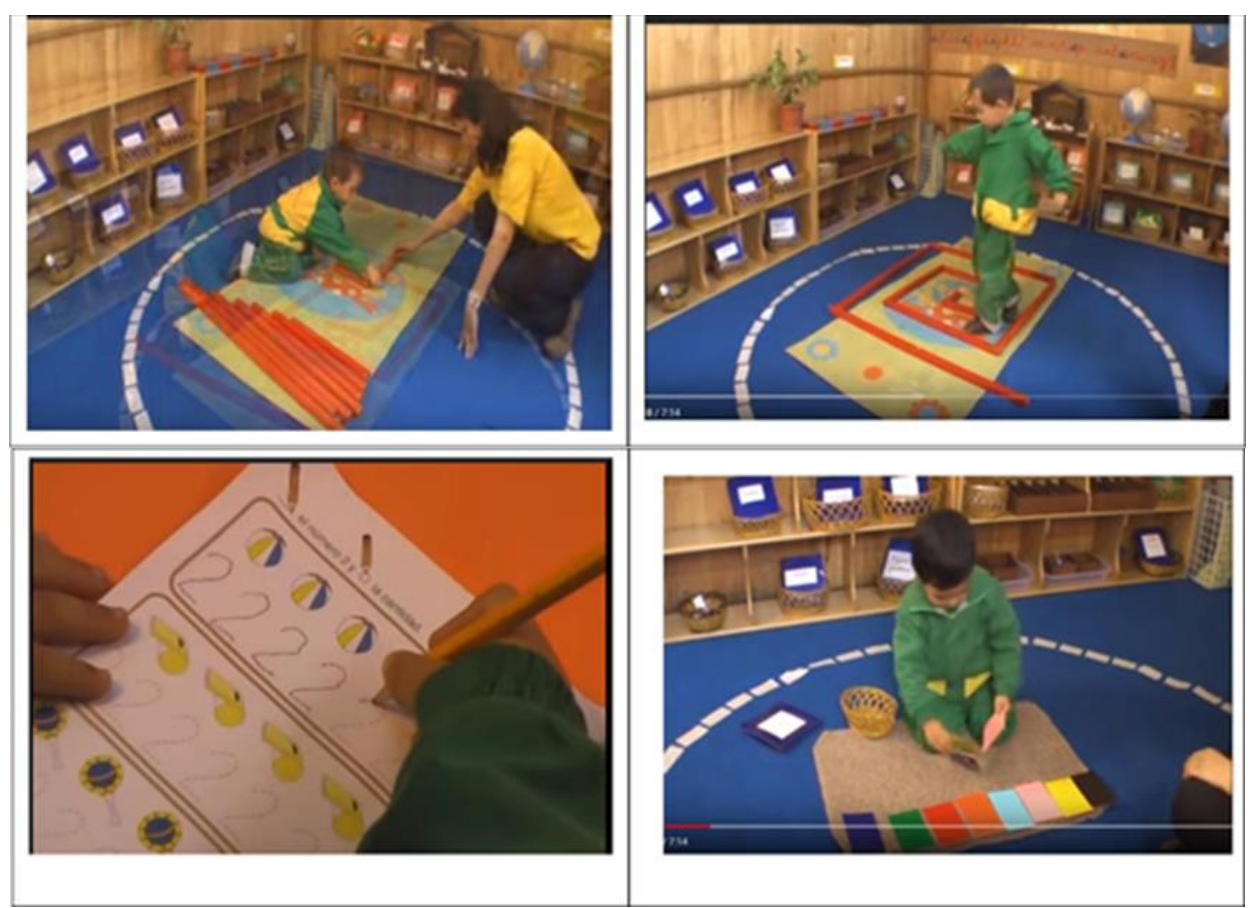

Se aplicará una metodología ecléctica en el desarrollo de la temática, como es la teoría de Montessori, la teoría de Piaget, pero convertida en una pedagogía aplicada, transformada.

\section{Marco Teórico}

\section{Rehabilitación Básica Comunitaria}

La realidad biológica de cada ser humano, desarrollada muchas veces al azar es lo que hace posible su existencia real. A partir de las diferencias de realidades los seres humanos comienzan a ser diferente a los demás y con ellos se analizan algunos síndromes y patologías reales, que la educación debe contribuir a su inclusión pero a su vez se necesita la existencia de modelos que permitan aplicar una pedagogía aplicada.

La aplicación de esa pedagogía amerita la utilización de modelos de rehabilitación básica que logre que el aprendizaje de aquellos que contienen elementos merecedores de una pedagogía de este tipo, logre que ellos sepan qué elementos utilizar cuando y donde. Esa rehabilitación debe estar cimentada en una estructura coherente basada en la experiencia docente y se ha de fundamentar en una base científica, cultural y pedagógica (Ehlers \& Gillberg, 1993).

La separación entre lengua y habla en la rehabilitación adquiere enorme importancia, por cuanto son muchos los fenómenos que provocan que sean diferenciables en ellos la semántica y la pragmática de la lengua. En la medida que las personas logren llevar a los significados, los docentes deben saber en qué procesos de adquisición del lenguaje, la metáfora o la metonimia son posibles o no en los niños. Las metáforas deben ser medidas experimentalmente en diferentes niños. El caso de los niños con insomnio por hiperactividad, el jardín se tiene que convertir en un lugar propicio en el que se pueda construir los ámbitos a partir de música como la 
del Centro Psicológico Integral, 2014. En este vídeo los autores crean la música adecuada que va a llenar la inteligencia musical del niño, y va a hacer posible una convivencia pacífica.

\section{Rehabilitación Básica Comunitaria e Innovación artística}

El año 2013 Televisión Española entrevista a la Dra. Isabel Dziobek, neuropsicóloga de la Universidad Libre de Berlín, acerca de la forma como ellos estaban manejando el autismo, desde la niñez. Para ella, en la entrevista realizada, los autistas poseen una coordinación menor a nivel cerebral. (Dziobek, 2013) para ella, el autismo es un trastorno de la empatía. En el caso del baile que bien se puede implementar en la rehabilitación, ellos tienen problemas para imitar los movimientos físicos.

Por eso es necesario el utilizar la danza y el baile para la coordinación. Un análisis de perfil del movimiento para saber si el cerebro de ellos puede además de repetir movimientos, coordinarlos con el de los demás. Hay que concebir el baile o la danza como un deporte. Otro proceso necesario es el de la dieta que se recomienda con los niños con autismo. Hace más o menos hace 20 años uno de cada 120 niños tenía autismo, pero ahora se tiene que de cada 60 niños, uno sea autista, y algo está pasando para que esos niños sean autistas. En la Universidad Académica de Chile, la profesora en la Escuela de danza UAHC, realizó un estudio en relación a Danza para los ojos azules, en la que se establece que (Vergara, 2016)

Danza para los ojos azules es producto de un proceso creativo llevado a cabo en el año 2014 con estudiantes de distintas generaciones de la Escuela de danza Espiral y la Universidad Academia de Humanismo Cristiano en Santiago de Chile. Dicho proceso tuvo como propósito hacer conexión entre cuerpo, espacio y memoria valorando el sentido educativo y colectivo de la danza reunida en diversidad y riqueza corporal, la cual desde su armonía y poética danza en un ritual de celebración de la vida y el renacer constante, en espiral, de la Maestra Joan Jara, origen de este vuelo creativo. (Pág. 28)

Una de las manifestaciones artísticas que puede permitir el desarrollo del niño en diferentes ámbitos, está el hecho de llegar a hacer teatro. Lo que les permite asumir diferentes roles, y expresarse teatralmente ya sea en una narración o en un poema. El niño aprende a seguir reglas en el vestir y en la expresividad. Según (Martínez, 2016) "El teatro es un arte total y como tal puede integrarse y compaginarse con cualquier otra manifestación, sea cual sea su naturaleza." (Pág. 5) Por lo tanto en ese proceso de socialización el arte teatral sirve para que ellos incorporen la inteligencia social a sus vidas y al lenguaje, que tanto les cuesta llegar a esos estados.

\section{Epistemología de la rehabilitación comunitaria infantil y sus escenarios}

La interpretación de los verdaderos escenarios que deben cubrir la vida de los niños con necesidades educativas especiales es vital para su abordaje. A partir de que el conocimiento es un proceso que se desarrolle por fases diferentes y que muchas veces es diferente en el sujeto cognoscente, a través de esta investigación se trata de analizar cómo conoce el arte el niño de tres a siete años, los niños con Asperger, los autistas, los hiperactivos y qué elementos se deben incorporar a su aprendizaje. 
En el área del lenguaje como elementos reales se estimulan los sentidos y se incrementa el vocabulario y que ellos ya puedan representar en tarjetas y percibe con sus sentidos, las dimensiones, volúmenes, formas y color, estableciendo un orden matemático y geométrico. Para llegar a ese estado las estrategias metodológicas deben ser organizadas para que los materiales lleguen al aula como resultado de una buena gestión de los recursos.

Un niño autista, es uno de los que más tiene contacto con los padres, por el trastorno que padece. En su comunicación no hacen la deipsis (señalar con el dedo) es decir epistemológicamente el subjetivismo es la escuela epistemológica que está más presente en ellos. El subjetivismo puede ser idealista. El subjetivismo idealista los hace únicos. Por lo que en el mundo que vive es importante no gritarles a los niños. Oye, pero no interpreta el mensaje que se le dice, por lo que es importante desarrollar en ellos las imágenes y las representaciones. Habría que analizar cuán importante puede ser el uso de la tecnología en el estímulo- respuesta de ellos.

El año 2012, en el Centro de Salud San Martín de Vitoria en Bilbao- España, se hizo un estudio que tenía como finalidad, llegar a establecer los parámetros del trastorno de atención con hiperactividad en el que se incluían a la familia como informante del TDAH, (García, 2012)

Es considerado como una patología multifactorial, en lo que respecta a su etiología y como consecuencia del determinismo genético se enmarca en la noción de vulnerabilidad genética, es decir, los factores genéticos se limitan a incrementar el riesgo de la expresión fenotípica modificando la expresión clínica del trastorno. (Pág. 7)

Si bien en la hiperactividad, se puede dar el caso del determinismo genético, es importante también que se logre manejar de la mejor manera esta problemática a partir del arte. El collage para el autista debe ser analizado, cómo el collage puede informar al autista tomando en cuenta su memoria visual, tal como aparecería en los niños con el síndrome de Asperger.

\section{Innovación estética}

La innovación artística es la creación de procesos que acompañan al ser humano a partir de la belleza del arte. En la innovación artística la parte motriz debe involucrar una lentitud diferente, al tener retrocesos en la parte cognitiva para los niños con autismo. El uso de la creatividad en forma divertida, los niños deben desarrollarlo en un buen entorno en el orden y la disciplina. Ante la necesidad de manipular objetos y ante los periodos sensibles del orden los niños deben aprender a ser organizados e independientes, para que desarrolle sus habilidades y destrezas motoras como es el caso de la manipulación de objetos que ayude al enriquecimiento de los sentidos. Para administrar se debe ayudar al orden, la disciplina y ayudar al niño a mejorar su concentración.

\section{Resultados}




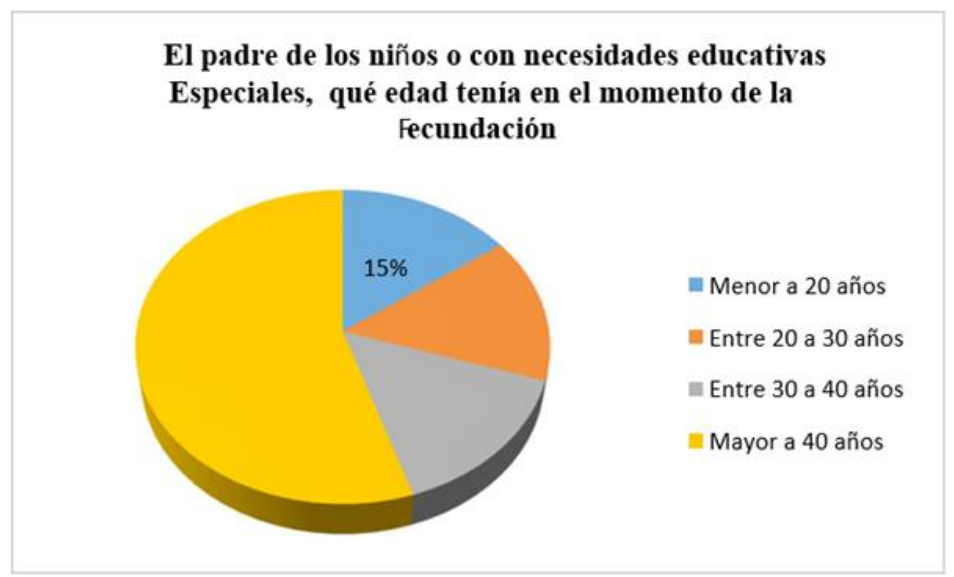

Figura 1

En la figura 1 encontramos que de los niños encuestados, la edad de los padres tienen una influencia ya que la más del $50 \%$ de los padres con NEE han tenido más de 40 años al momento de la fecundación por la unión del espermatozoide con su óvulo.

En la figura 2 tratamos de entender los antecedentes familiares de personas con niños con NEE para ello en la escuesta se preguntó a) El padre trabajó en una planta industrial con manipulación de químico. b) Los padres viven o vivían cerca de una planta industrial, en la que respira los desechos tóxicos. c) Usted como madre de familia ha trabajado en una fotocopiadora estando embarazada. d) Usted tuvo hijos a la edad de mayor de 60 años. e) Usted tuvo su primero y segundo hijo siendo mayor de 40 años

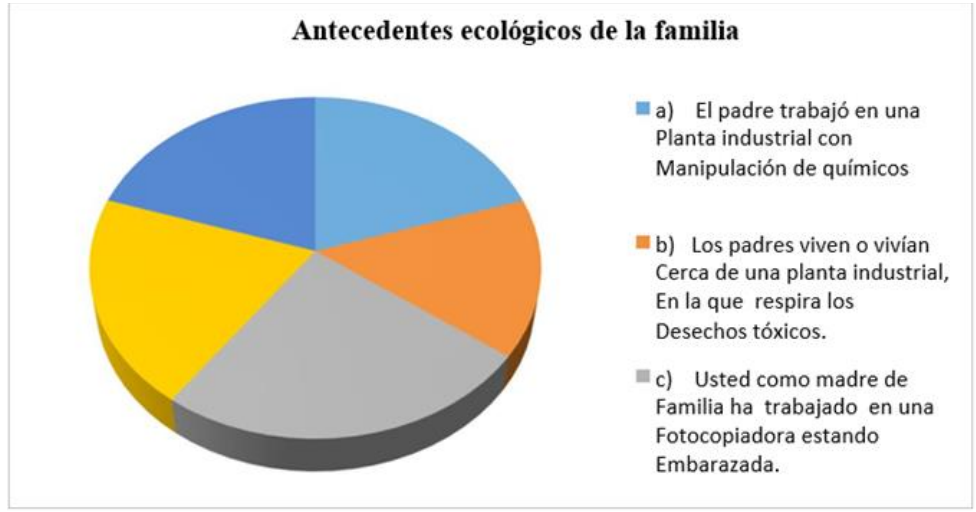

Figura 2

De la figura encontramos que la mayoría de madres de familia trabajan cerca de una maquina fotocopiadora. El $15 \%$ de los padres trabajaban cerca de una planta industrial y el $20 \%$ de los padres trabajaron con manipulación de químicos. Ambos padres de alguna forma fueron influenciados de alguna tipo de químico o elemento toxico.

En la figura 3 podemos ver la tendencia de gusto de los niños entre las opciones se le pregunto entre la música, los números o el deporte. 


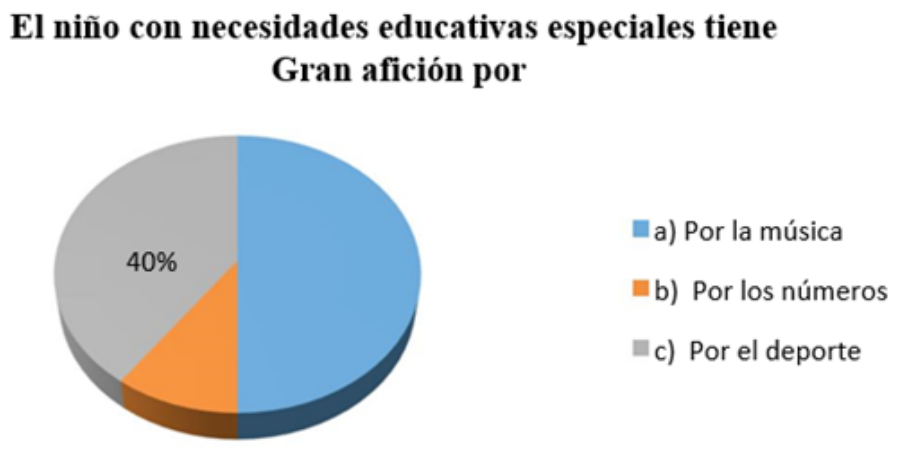

Figura 3

De lo cual vale recalcar que la mitad de la población demostró una tendencia hacia la música y un $40 \%$ hacia los deportes. Rompiendo el prejuicio de que niños con necesidades no pueden realizar deportes.

La figura 4 se enfoca en la perspectiva de cuál debería ser el comportamiento de un niño con necesidades al estar en un evento público. Entre las opciones dadas a las personas se les dio. a) Se aísla, b) Socializa con personas de su edad, c) Juega solo o d) Socializa con personas de todas las edades. Entre las respuesta encontramos que el $60 \%$ dijo que los niños se aíslan; mientras que el otro porcentaje significante respondió que ellos juegan solo.

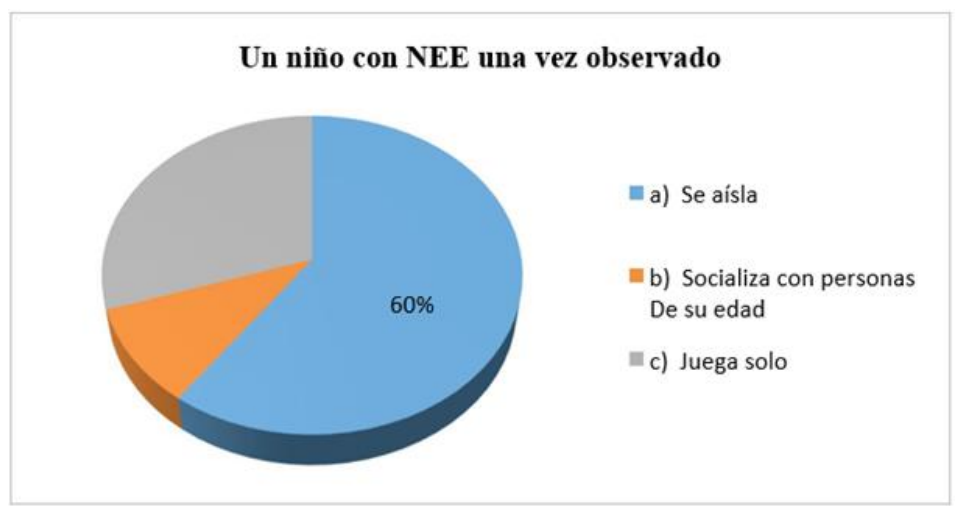

La figura 5 pregunta se enfoca hacia el comportamiento cotidiano de un niño con N.E.E. entre las opciones se les dio a) Tiende su propia cama, b) Recoge sus platos una vez que ha terminado de comer, c) Se lava la boca y dientes de forma autónoma y d) Respeta los turnos para ir al baño. Se encontró que los niños con NEE son capaces de cumplir varias funciones al igual que todo niño normal entre ellas tenemos el respetar el turno al baño, lavarse la boca, entre otros. 


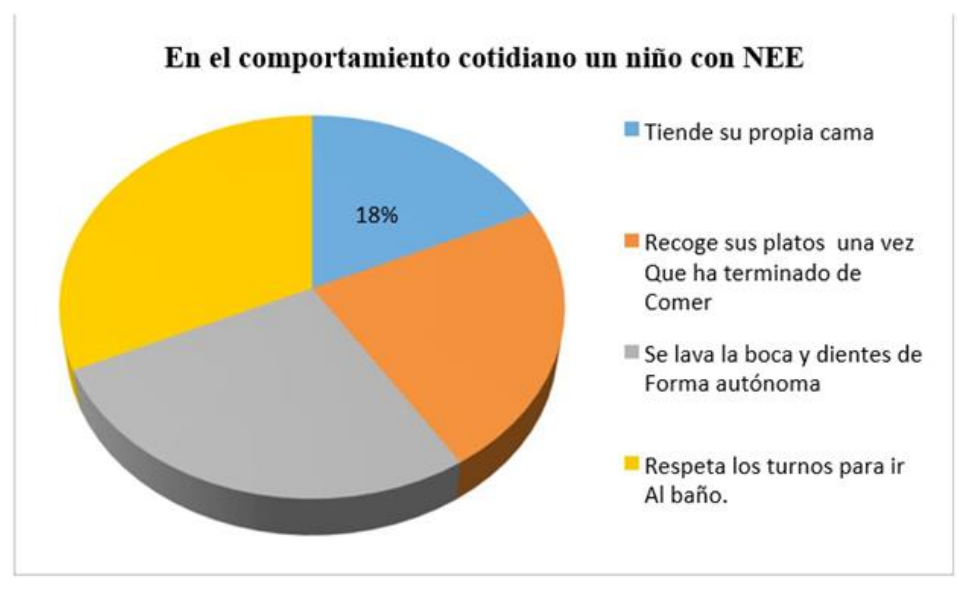

\section{Conclusiones}

La estabilización de los procesos de niños con necesidades educativas especiales se puede realizar a través del análisis de dos postulados genéticos, el del sistema cromosómico o naturalista y el del aprendizaje a través de la teoría de la genética según Piaget. Así como la naturaleza tiene sus leyes, así también las NEE las tienen, por lo que es importante llegar a formar pautas de rendimiento, de acuerdo a sus posibilidades no a las pautas preestablecidas.

El esfuerzo de los padres con NEE debe ser cofinanciada por el estado, con la finalidad de llegar a establecer qué aspectos merecen. Ninos con NEE son capaces de alcanzar tantos logros, también pueden obtener independencia para su cuidado y aseo personal. Niños con NEE tiene pasión por la música y aun se demostró que muchos de ellos aman el deporte.

Es importante seguir estudiando y aprendiendo de niños con NEE para que la educación sea flexible y aceptable a sus necesidades.

\section{Bibliografía}

Attwood, T. (2007). What is Asperger's Syndrome. Retrive from: www. tonyattwood.com. Consultado.

Blanco, A. (2016). El teatro como herramienta socializadora para personas con Asperger. Reop .

Centro Psicológico Integral . (2014, 03 07). Música Para Dormir Bebes Niños Hiperactivos Insomnio Infantil. Retrieved From Música Para Dormir Bebes Niños Hiperactivos Insomnio Infantil: https://www.youtube.com/watch?v=DGXyYvkJLKE

Dziobek, D. I. (2013, 03 25). Música para tratamiento del autismo. Retrieved from Visión futuro : https://www.youtube.com/watch?v=d619xp0tUno

Ehlers, S. \& Gillberg, C.(1993). The Epidemiology of Asperger Syndrome. A total Population Study. Journal of Child Psychology and Psychiatry 1993; 34:8, 1327-1350.

Fernández, M. (2013: pág. 43). Creatividad, arte terapia y autismo. Arte, individuo y sociedad. 
García, J. (2012). La familia como informante del TDAH: Convergencia de las fuentes y efecto de los antecedentes familiares. Boletín S. Vasco Pichate.

Hall K. (2003). Soy un niño con síndrome de Asperger, Barcelona, Paidós, 2003.

Martínez, B. (2016). El teatro como herramienta socializadora para personas con Asperger. Aeop: Asociación Española de Orientación y Psicopedagogía.

Romanowski P. \& Kirby, B.A. (2005). The OASIS to Asperger syndrome. New Cork, Crown Publishers.

Szatmari P. (2006). Comprender a los niños con autismo y síndrome de Asperger. Barcelona: Paidós,

Thomas I. G. (2003). El síndrome de Asperger: Estrategias prácticas para el aula. Guía para el profesorado. País Vasco, Servicio Central de Publicaciones.

Vergara, Y. (2016). Danza para los ojos azules. Revista de Estudios de Sociedad.

Williams K. (1995). Comprender al estudiante con síndrome de Asperger. Orientación para profesores. Focus on autistic behavior, 10, (2): PRO-ED, Inc.

Wing L. (1981). Asperger's syndrome: a clinical account. Psychological Medicine. 11: 115-130. 